\title{
Efecto de la adición de bentonita sobre las propiedades ópticas de diferentes materiales compuestos poliméricos
}

\section{Effect of bentonite addition on the optical properties of different polymeric composites materials}

\author{
María Gabriela Passaretti ${ }^{1,2}$, Mario Daniel Ninago ${ }^{1,3}$, Olivia Valeria López ${ }^{1}$, \\ Andrés Eduardo Ciolino ${ }^{1,2}$, Daniel Alberto Vega ${ }^{4,5}$, \\ Marcelo Armando Villar ${ }^{1,2}$
}

\footnotetext{
${ }^{1}$ Planta Piloto de Ingeniería Química, PLAPIQUI (UNS-CONICET), CP: 8000, Camino “'La Carrindanga” Km 7, Bahía Blanca, Buenos Aires, Argentina.

${ }^{2}$ Departamento de Ingeniería Química, Universidad Nacional del Sur, CP: 8000, Av. Alem 1253, Bahía Blanca, Buenos Aires, Argentina.

${ }^{3}$ Facultad de Ciencias Aplicadas a la Industria, Departamento de Ingeniería Química, Universidad Nacional de Cuyo, CP: 5600, Bernardo de Irigoyen 375, San Rafael, Mendoza, Argentina.

${ }^{4}$ Instituto de Física del Sur, IFISUR (UNS-CONICET), CP: 8000, Av. Alem 1253, Bahía Blanca, Argentina.

Facultad de Ciencias Aplicadas a la Industria, Departamento de Ingeniería Química, Universidad Nacional de Cuyo, CP: 5600, Bernardo de Irigoyen 375, San Rafael, Mendoza, Argentina.

${ }^{5}$ Departamento de Física, Universidad Nacional del Sur, CP: 8000, Av. Alem 1253, Bahía Blanca, Buenos Aires, Argentina.

e-mail: mgpassaretti@plapiqui.edu.ar,mninago@plapiqui.edu.ar, olivialopez@plapiqui.edu.ar, aciolino@plapiqui.edu.ar, dvega@uns.edu.ar, mvillar@plapiqui.edu.ar
}

\begin{abstract}
RESUMEN
En este trabajo se obtuvieron materiales compuestos a partir de la incorporación de bentonita a matrices poliméricas biodegradables y sintéticas. Se empleó una muestra de bentonita comercial (BE) y una natural (BM), además de tres matrices poliméricas diferentes: almidón de maíz termoplástico (TPS), polietileno de alta densidad (HDPE) y un copolímero tribloque, poli (estireno- $b$-butadieno- $b$-estireno) (SBS). Los materiales compuestos se obtuvieron incorporando $5 \% \mathrm{w} / \mathrm{w}$ de carga y se procesaron por mezclado en fundido. Películas de las distintas formulaciones se obtuvieron mediante termo-compresión en una prensa hidráulica. Su apariencia y homogeneidad se estudió mediante Microscopía Electrónica de Barrido (SEM). La opacidad y la capacidad de barrera a la radiación UV se determinaron a partir de los espectros de absorbancia obtenidos en un espectrofotómetro. Las mediciones de color se realizaron utilizando un colorímetro en modo transmitancia, registrándose los parámetros $L, a$ y $b$. En las micrografías SEM, se observó una buena distribución de la carga en las tres matrices poliméricas. La adición de bentonita modificó las propiedades ópticas de las películas debido al efecto bloqueante de las mismas, lo cual se evidenció en un incremento en la capacidad de barrera a la radiación UV y en la opacidad de todos los materiales compuestos estudiados.
\end{abstract}

Palabras clave: bentonita; compuestos poliméricos; microestructura; propiedades finales

\section{ABSTRACT}

In this work, composite materials were obtained by the incorporation of bentonite to biodegradable and synthetic polymeric matrices. Commercial (BE) and natural (BM) bentonite samples were employed, as well as three different polymeric matrices: thermoplastic corn starch (TPS), high density polyethylene (HDPE) and a poly(styrene- $b$-butadiene- $b$-styrene) triblock copolymer (SBS). Composite materials were obtained by incorporating $5 \% \mathrm{w} / \mathrm{w}$ filler by melt mixing. Films were obtained by thermo-compression in a hydraulic press. By Scanning Electron Microscopy (SEM) it were examined the homogeneity and appearance of films. Opacity 
and UV barrier capacity were determined from the UV-vis spectra recorded in a spectrophotometer. Film color measurements were performed using a colorimeter in the transmittance mode, recording parameters $L$, $a$, and $b$. From SEM micrographs, a good filler distribution in the three different matrices were observed. The addition of bentonite modified the optical properties of films due to their blocking effect, evidenced in an increase in both UV barrier capacity and opacity.

Keywords: bentonite; polymeric composites; microstructure; final properties.

\section{INTRODUCCIÓN}

Las bentonitas son arcillas constituidas principalmente por montmorillonita, mineral que pertenece al grupo de las esmectitas dioctaédricas. Las bentonitas son filosilicatos laminares con estructura 2:1 ya que presentan una capa octaédrica de alúmina entre dos capas tetraédricas de sílice [1-3]. Además, pueden estar compuestas por otros componentes tales como cuarzo, feldespato, carbonatos, y óxidos de metales [4,5]. Poseen un amplio rango de aplicaciones debido principalmente a sus propiedades coloidales y de hinchamiento, como así también, a su carácter plástico cuando se mezclan con líquidos polares como el agua [5, 6]. El interés científico e industrial en el uso de arcillas naturales radica en su abundancia a nivel mundial, fácil extracción y bajo costo [7]. Por ejemplo, las bentonitas se emplearon como adsorbentes, en sistemas de intercambio de iones, y como catalizadores [8-11].

La incorporación de partículas minerales en matrices poliméricas permite mejorar su estructura y las propiedades mecánicas y de barrera de los compuestos. Las bentonitas cumplen con los principales requisitos para ser empleadas en aplicaciones poliméricas ya que son abundantes, económicas, prácticamente incoloras, inertes y sus partículas tienen la forma y el tamaño como para transformarlas en una opción lógica como relleno alternativo para producir materiales compuestos de bajo costo. Antes de incorporar bentonita a las diferentes matrices, el mineral debe someterse a una serie de procesos que incluyen secado, trituración, purificación, y clasificación [12]. La presencia de impurezas comunes tales como calcita, feldespato, cuarzo, mica y materia orgánica, tienen un efecto adverso sobre la estabilidad térmica y la capacidad de intercambio catiónico de las bentonitas. Por consiguiente, tanto para obtener mejores propiedades físicas, mecánicas y térmicas, como para encontrar óptimas aplicaciones de envasado, resulta esencial su etapa de purificación previa.

La preparación de las arcillas minerales involucra una serie de procesos físicos y químicos que tienen por objeto potenciar sus propiedades para aplicaciones industriales específicas [13]. La modificación de sus propiedades de superficie mediante tratamientos de distinta naturaleza (ácidos, térmicos o pilarización), o bien de modificación de su espaciado interlaminar tienen gran importancia desde el punto de vista industrial. Según ROHLMANN et al. [14] y MAGALHÃES y ANDRADE [15], la potencialidad de la bentonita como agente de relleno radica en la capacidad de las mismas para dispersarse homogéneamente dentro de la matriz, mejorando así las propiedades finales de los compuestos. De acuerdo a lo expuesto por BERGAYA y LAGALY [1], la posibilidad de desarrollar compuestos formados por una base polimérica y partículas minerales como agentes de refuerzo permitiría diseñar nuevos materiales con propiedades termo-mecánicas superiores. La adición de minerales a polímeros permite obtener diferentes tipos de materiales compuestos (intercalados, exfoliados, o ambos), en función de la organización de las partículas en la matriz. Por otra parte, la morfología o estructura de los compuestos depende no sólo de la compatibilidad entre las distintas fases sino también del método de mezclado y de su dispersión. En la preparación de materiales compuestos polímero/bentonita se han utilizado una amplia variedad de polímeros termoplásticos, principalmente poliolefinas, estirénicos, etc., y también materiales termorrígidos, tales como resinas epoxi y compuestos fenólicos [16].

La literatura reporta diferentes métodos de preparación de materiales compuestos tales como polimerización in situ, intercalación inducida por solución, y procesamiento en fundido, entre otros. Entre las mejoras observadas en las propiedades finales se destacan una mayor resistencia y módulo elástico, la disminución de la inflamabilidad, y mayores estabilidades térmicas y propiedades de barrera. Así, los cambios en las propiedades térmicas, mecánicas, e inflamabilidad han dado lugar a aplicaciones en la industria automotriz, entre otras. Por otra parte, las propiedades de los materiales compuestos cambian significativamente a medida que las dimensiones de las partículas empleadas como relleno se reducen a dimensiones nanométricas. Un bajo nivel de carga conduce a que la densidad total del compuesto sea similar a la del polímero puro, facilitando su potencial de procesamiento para la obtención de películas o fibras [17].

En este trabajo se emplearon bentonitas comerciales y naturales como agentes de relleno de tres matrices poliméricas: almidón termoplástico, polietileno de alta densidad, y poli(estireno- $b$-butadieno- $b$ estireno). Los materiales finales se caracterizaron estructuralmente y se evaluó el efecto de la bentonita sobre las propiedades ópticas de los compuestos. 


\section{MATERIALES Y MÉTODOS}

\subsection{Materiales}

Se emplearon tres matrices poliméricas diferentes: almidón de maíz termoplástico (TPS), polietileno de alta densidad (HDPE) y un copolímero tribloque poli(estireno-b-butadieno-b-estireno) (SBS). Para la obtención del TPS se usó almidón de maíz nativo provisto por Misky-Arcor (Tucumán, Argentina) y como plastificantes se empleó glicerol (Anedra) y agua destilada. Se trabajó con un HDPE comercial (NG7000, Dow-Polisur S.A.) con un índice de fluidez de 11 a $190{ }^{\circ} \mathrm{C} / 21,6 \mathrm{Kg}$ ASTM 1238. El SBS (Sigma Aldrich) utilizado presenta una masa molar promedio en peso $\left(\mathrm{M}_{\mathrm{w}}\right)$ de $140,000 \mathrm{~g} \mathrm{~mol}^{-1}$ con un $30 \% \mathrm{w} / \mathrm{w}$ de estireno.

La bentonita comercial (BE) y natural (BM) fueron suministradas por Gabriel Bouillard \& Cia (Argentina). Las muestras minerales se trituraron para reducir el tamaño inicial de las partículas. Luego se tamizaron empleando tamices Zonytest (ASTM $\mathrm{N}^{\circ} 40$ ) para descartar el material no arcilloso y obtener partículas con diámetros medios inferiores a $420 \mu \mathrm{m}$.

\subsection{Caracterización de las partículas minerales}

La morfología de la bentonita comercial y natural se estudió mediante Microscopía Electrónica de Barrido (SEM), empleando un microscopio electrónico JEOL JSM-35 CF, con detector de electrones secundarios a $10 \mathrm{kV}$. Las muestras fueron previamente dispersadas, empleando un flujo de aire, sobre una cinta adhesiva conductora de aluminio $\left(3 \mathrm{M}^{\circledR}\right)$ y recubiertas con una capa de oro mediante sputtering $(\sim 30 \AA)$.

La distribución de tamaños de las partículas de BE y BM se determinó mediante Difracción Laser (LD) empleando un equipo Horiba Partica LA-950.

\subsection{Preparación de las mezclas y obtención de las películas}

Compuestos TPS-BE y TPS-BM: se procesaron en fundido mezclas de almidón, glicerol (30\% w/w, base almidón), agua ( $45 \%$ w/w, base almidón) y BE ó BM (5\% w/w, base mezcla) en una mezcladora Atlas Laboratory a $140{ }^{\circ} \mathrm{C}$ y $50 \mathrm{rpm}$ durante $15 \mathrm{~min}$. Las mezclas procesadas se trituraron y acondicionaron a $25^{\circ} \mathrm{C}$ y $60 \%$ de humedad relativa (HR). Las películas se obtuvieron a partir de las mezclas acondicionadas mediante termo-compresión en una prensa hidráulica a $140{ }^{\circ} \mathrm{C}$ y $180 \mathrm{~kg} \mathrm{~cm}^{-2}$ durante 6 min.

Compuestos HDPE-BE y HDPE-BM: se procesaron en fundido mezclas de HPDE y BE ó BM (5\% w/w), empleando una mezcladora Atlas Laboratory a $160^{\circ} \mathrm{C}$ y $40 \mathrm{rpm}$ durante $15 \mathrm{~min}$. Las películas se obtuvieron mediante termo-compresión en una prensa hidráulica a $160{ }^{\circ} \mathrm{C}$ y $180 \mathrm{~kg} \mathrm{~cm}^{-2}$ durante 6 min.

Compuestos SBS-BE y SBS-BM: Se procesaron en fundido mezclas de SBS y BE ó BM (5\% w/w), empleando una mezcladora Atlas Laboratory a $140{ }^{\circ} \mathrm{C}$ y $50 \mathrm{rpm}$ durante $15 \mathrm{~min}$. Las películas se obtuvieron mediante termo-compresión en una prensa hidráulica a $140{ }^{\circ} \mathrm{C}$ y $80 \mathrm{~kg} \mathrm{~cm}^{-2}$ durante $5 \mathrm{~min}$.

\section{CARACTERIZACIÓN DE LAS PELÍCULAS}

\subsection{Caracterización estructural}

La distribución de las partículas en las matrices se estudió mediante Microscopía electrónica de barrido (SEM), evaluándose además la homogeneidad y apariencia de los materiales compuestos. Se empleó un microscopio electrónico JEOL JSM-35 CF, con detector de electrones secundarios. Las películas fueron criofracturadas y se recubrieron con una capa de oro mediante sputtering $(\sim 30 \AA)$.

La estructura cristalina y el grado de intercalación de los materiales compuestos se estudiaron mediante Difracción de Rayos X (XRD) empleando un difractómetro Philips PW1710, provisto de un detector operando a $45 \mathrm{kV}$ y $30 \mathrm{~mA}$ en un rango $2 \Theta$ desde $3 \mathrm{a} 60^{\circ}$. El espaciado basal entre las láminas de bentonita $d$ se determinó de acuerdo a la ecuación de Bragg [18].

\subsection{Propiedades ópticas}

La capacidad de barrera a la radiación UV y la opacidad de las películas se determinaron por Espectroscopía UV-vis. Se obtuvieron los espectros de absorción entre 200 y $800 \mathrm{~nm}$ en un espectrofotómetro Shimadzu UV-160, empleando una cubeta de cuarzo. La opacidad de las películas se calculó a partir del área bajo la curva registrada entre 400 y $700 \mathrm{~nm}$, de acuerdo al método reportado por PIERMARÍA et al. [19]. Las muestras se analizaron al menos por triplicado. 
El color de las películas se determinó en un colorímetro Hunterlab UltraScan XE en modo transmitancia. Se registraron la luminosidad $(L)$ y los parámetros de cromaticidad $a$ (verde-rojo) y $b$ (amarillo-azul) de la escala Hunter. Se realizaron al menos 10 determinaciones en diferentes puntos al azar y se informaron los valores promedio de las mismas.

\section{RESULTADOS}

\subsection{Caracterización de las partículas minerales}

En la Figura 1 se muestran las micrografías SEM de las partículas minerales de bentonita comercial (BE) y natural (BM).
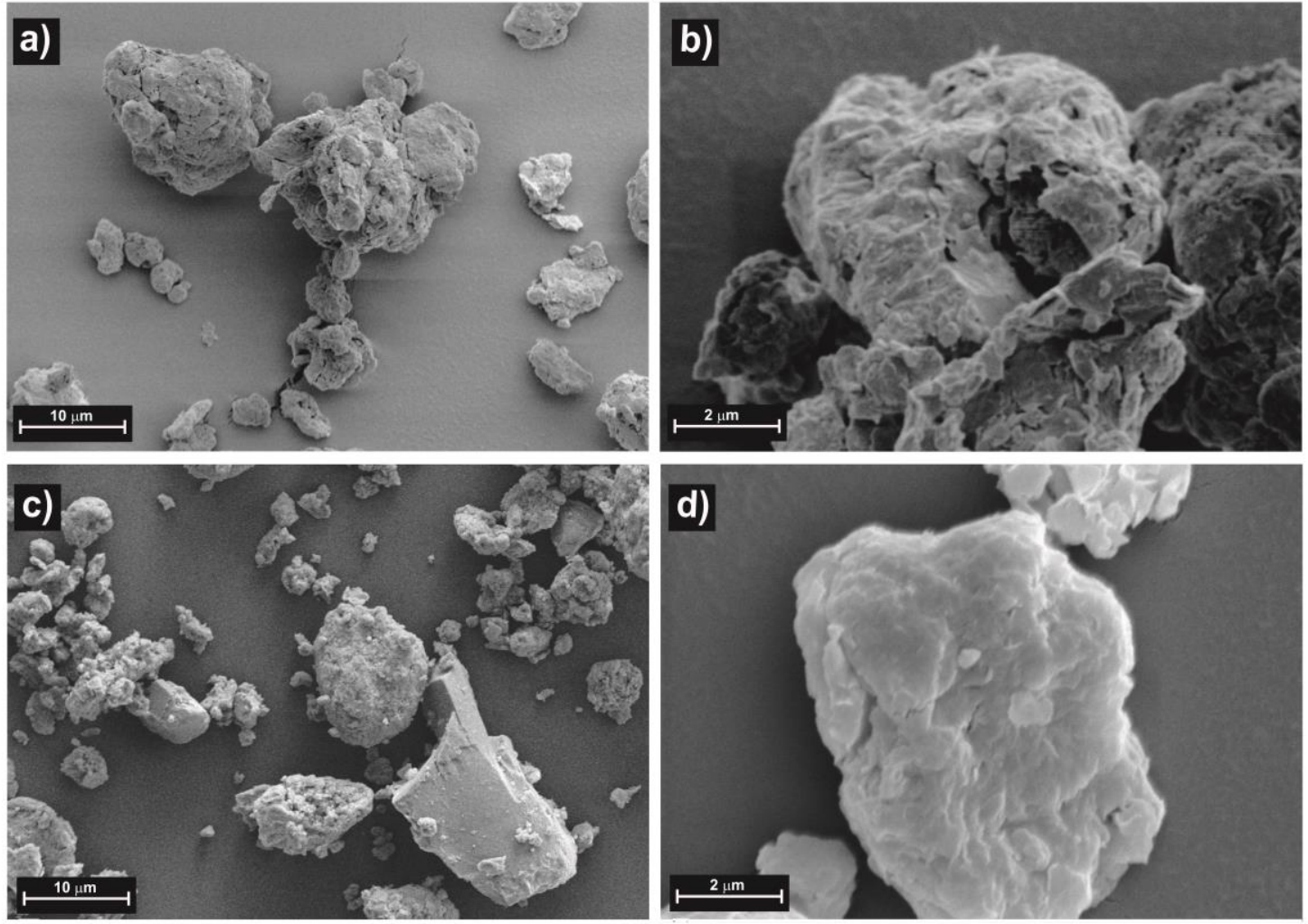

Figura 1: Micrografías SEM de partículas minerales de bentonita: a) y b) comercial (BE) y c) y d) natural (BM).

En ambas muestras se evidenció la presencia de dos poblaciones de agregados pseudo-esféricos con bordes irregulares (Figura 1a y 1c). En la muestra BE se detectaron agregados de partículas de tamaños medios de $\sim 3,5 \mu \mathrm{m}$ y $\sim 13,0 \mu \mathrm{m}$; mientras que para el caso de la muestra de BM se observaron dos poblaciones de $\sim 5,0 \mu \mathrm{m} \mathrm{y} \sim 13,0 \mu \mathrm{m}$. MODABBERI et al. [20] reportaron tamaños de partículas similares para diferentes bentonitas de origen iraní. En las Figuras 1c y 1d se observa que ambos minerales presentan una morfología típica de las arcillas conformadas por una estructura laminar en escamas [20]. Otros autores han reportado morfologías similares para diferentes partículas minerales [21,22]. La superficie de las partículas presentan poros, intersticios y cavidades. Dichas características morfológicas, típicas de las arcillas naturales, son las responsables de la alta capacidad de hinchamiento que poseen estos materiales [23-26].

La distribución de tamaño de partícula de las muestras minerales, determinadas mediante difracción láser, se muestra en la Figura 2. Para la BE se distingue una distribución de tamaños bimodal, evidenciándose partículas de $\sim 5,8 \mu \mathrm{m}$ y $\sim 35,2 \mu \mathrm{m}$. Asimismo, la muestra BM está constituida por dos poblaciones de partículas de tamaños similares a los de BE $(\sim 5,5 \mu \mathrm{m}$ y $\sim 39,8 \mu \mathrm{m}$, Figura $2 \mathrm{~b})$. Cabe destacar que, para ambas muestras, la proporción de partículas de menor tamaño representó más del $90 \%$ del total de la población. 


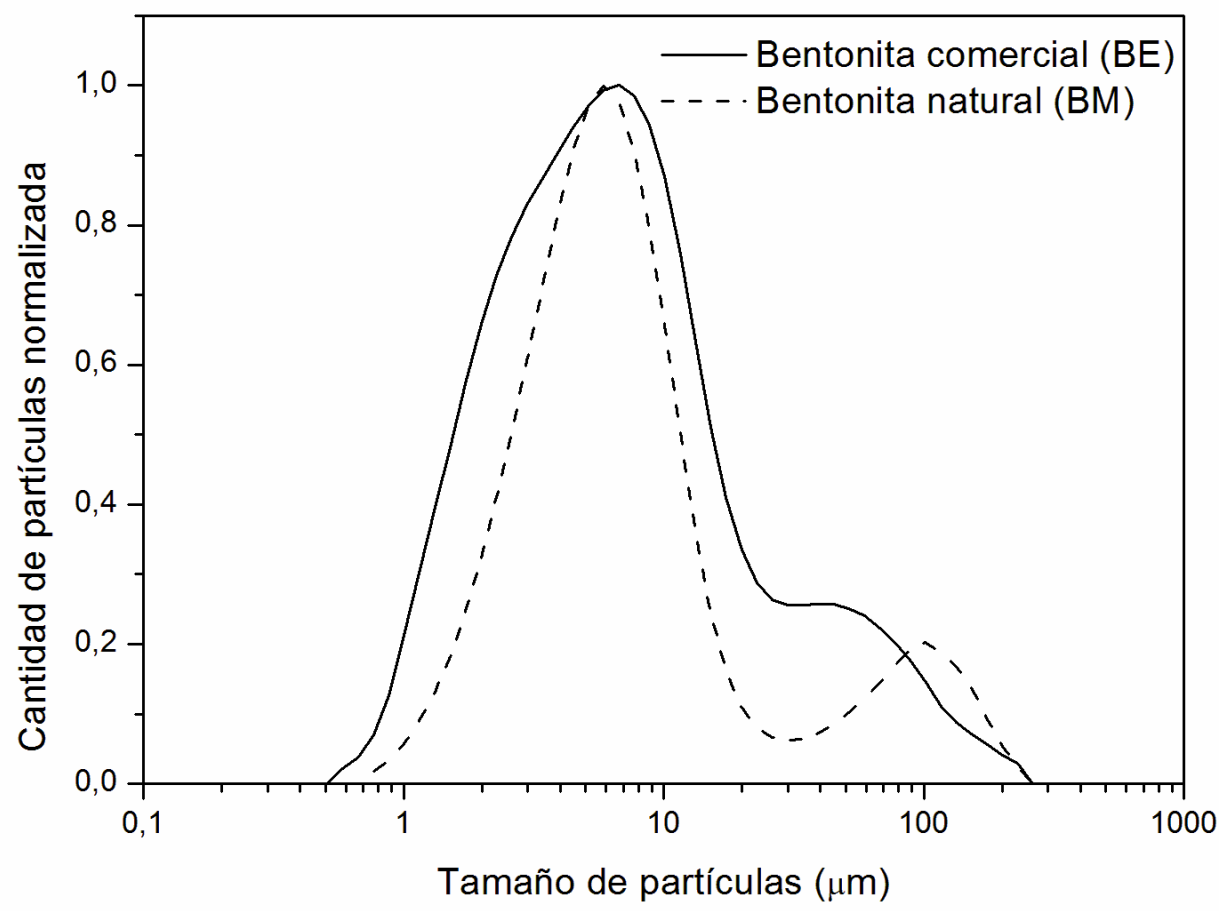

Figura 2: Distribución de tamaño por difracción láser (LD) de partículas minerales de bentonita comercial (BE) y natural (BM).

\subsection{Caracterización estructural de las películas}

La evaluación macroscópica de las películas obtenidas evidenció que, independientemente de la naturaleza de la matriz base y de la calidad de las partículas de bentonita, las mismas resultaron homogéneas, translúcidas, flexibles y fáciles de manipular.

El estudio de la microestructura de los materiales compuestos permite evaluar la homogeneidad de las matrices, la compatibilidad entre el relleno y la matriz y la dispersión de las partículas en el material final. La Figura 3 muestra micrografías SEM de los compuestos a base de TPS, HDPE y SBS conteniendo $5 \%$ w/w de BE ó BM.

Las superficies de fractura de las películas de TPS resultaron homogéneas y suaves, sin vestigios de gránulos de almidón no procesados. Además, el análisis de la microestructura tampoco evidenció la separación de las fases ricas en glicerol y en almidón, ni la presencia de canales de migración del plastificante hacia la superficie de las películas. Estos resultados podrían atribuirse a la eficiencia del procesamiento térmico. Por otra parte, la homogeneidad en el espesor de las secciones transversales de los materiales a base de TPS se debe a la eficacia del proceso de termo-compresión empleado para la obtención de las películas. La observación de las superficies de fractura de las películas de TPS cargadas con BE y BM evidenció la buena distribución de ambos rellenos en la matriz de almidón como resultado del óptimo mezclado en fundido de los compuestos. DE MELO et al. [25] también observaron superficies de fractura suaves con una buena distribución de partículas de arcillas en compuestos a base de almidón. La ausencia de aglomerados de partículas, potenciales concentradores de tensión que afectan el desempeño mecánico de los materiales, es otro aspecto positivo derivado del eficiente proceso de mezclado. La buena compatibilidad de BE y BM con el TPS permitió una buena adhesión de los rellenos minerales a la matriz de almidón, evitando su desprendimiento durante la crio-fractura de las películas.

Las secciones transversales de los materiales a base de HDPE resultaron suaves y homogéneas (Figura 3). En los materiales HDPE-BE/BM, si bien se evidenció una buena distribución de las partículas en la matriz 
sintética, se detectó la presencia de micro-aglomerados de bentonita, similares a los reportados por. SARIFUDDIN e ISMAIL [26]. Según SUHAIDA et al. [27] la forma irregular de la bentonita provee un área superficial suficiente que permite que el relleno se disperse bien y se fije de manera adecuada a la matriz. La formación de aglomerados podría relacionarse con el carácter hidrofílico de las partículas minerales en contraste con la naturaleza hidrofóbica de la matriz sintética. La ocurrencia de estos aglomerados conduce al desprendimiento de partículas durante la fractura del material, formándose así pequeños poros e intersticios. De acuerdo a SARIFUDDIN e ISMAIL [26], la expulsión o separación de estos aglomerados es indicativo de una pobre interacción entre la matriz y el relleno mineral. A partir de estos resultados, es esperable que el desempeño mecánico de las películas de HDPE se reduzca por la incorporación de partículas de bentonita sin modificar ya que estos aglomerados pueden actuar como puntos concentradores de tensión [28]. Esto podría evitarse mediante el uso de arcillas modificadas que mejorarían la afinidad entre la matriz y el relleno evitando efectos indeseados.

Los compuestos a base de SBS presentaron en todos los casos la estructura típica de matrices elastoméricas (Figura 3) con morfologías similares a las reportadas por LIETZ et al. [29] para copolímeros en bloque SBS reforzados con nanoarcillas. Este carácter se atribuye al elevado porcentaje de poli(butadieno) (70 \% w/w) que posee la matriz de SBS empleada en este trabajo. Particularmente, la irregularidad de la sección transversal del SBS puede relacionarse con la elasticidad típica de este tipo de materiales que dificulta su fractura vítrea. La técnica de procesamiento empleada permitió una adecuada distribución de las partículas de bentonita en la matriz sintética. Sin embargo, se observaron algunos aglomerados, como en el caso de los compuestos de HDPE. En las micrografías SEM se detectaron micro-poros, asociados a la pobre adhesión del relleno al SBS. La baja compatibilidad de la matriz de SBS con la bentonita sería la responsable del fenómeno de desprendimiento de las partículas durante la crio-fractura del material. LIAO et al. [30] sugieren como alternativa para mejorar la afinidad entre la bentonita y esta matriz sintética, el uso de partículas minerales modificadas. Estos autores reportaron mejoras significativas en la compatibilidad trabajando con partículas de montmorillonita organofílica. Asimismo, PEDRONI et al. [31] propusieron el procesamiento de los compuestos mediante extrusión para mejorar la adhesión de rellenos minerales a matrices de SBS.
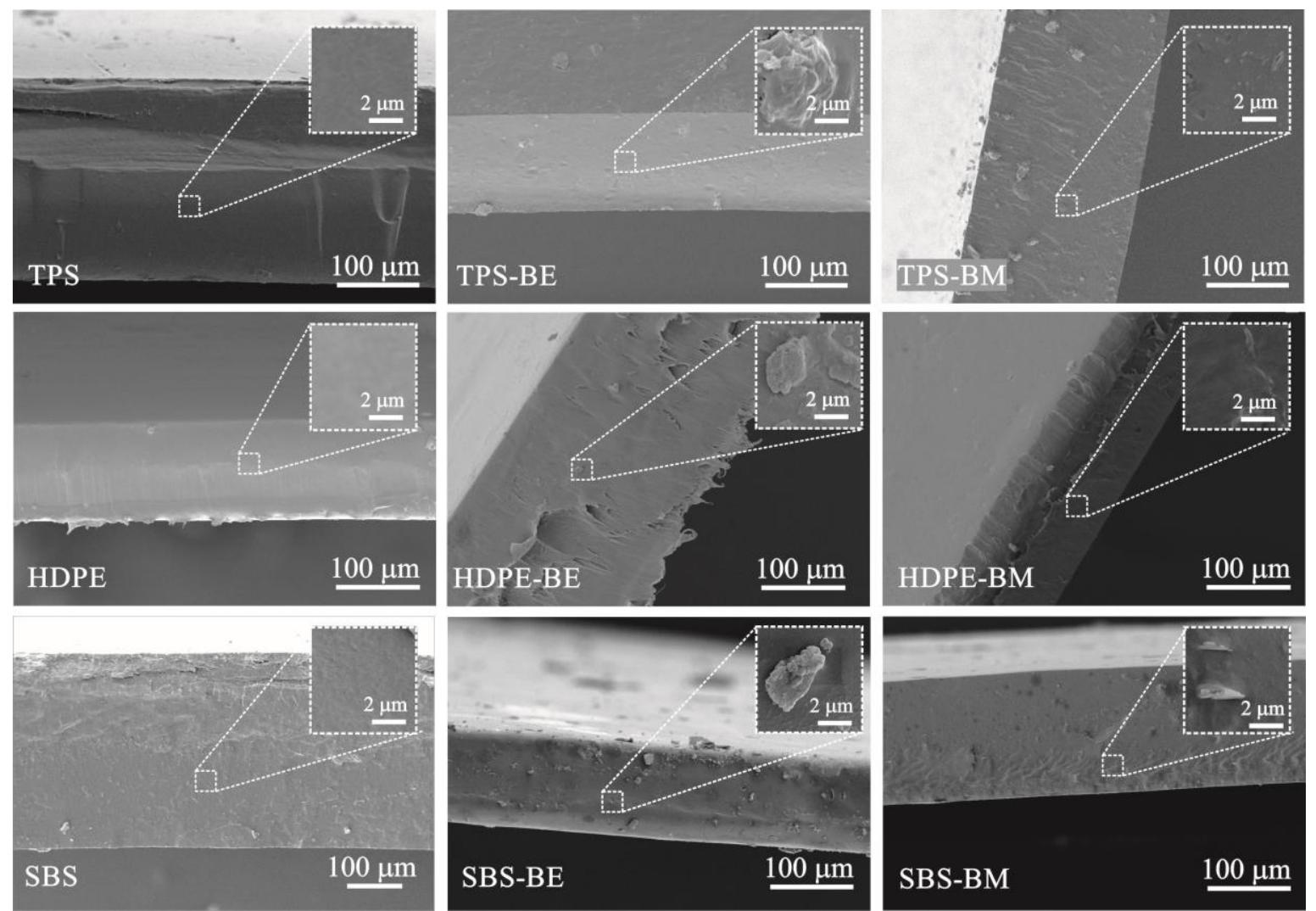

Figura 3: Micrografías SEM de compuestos a base de almidón termoplástico (TPS), polietileno de alta densidad (HDPE) y copolímero tribloque poli(estireno- $b$-butadieno- $b$-estireno) (SBS) y bentonita comercial (BE) ó natural (BM). 
La estructura cristalina de los materiales se estudió mediante Difracción de Rayos X, evaluándose el grado de intercalación de las cadenas poliméricas entre las láminas de las partículas de bentonita. En la Figura 4 se muestran los difractogramas de las muestras BE y BM, como así también de los compuestos obtenidos en este trabajo.
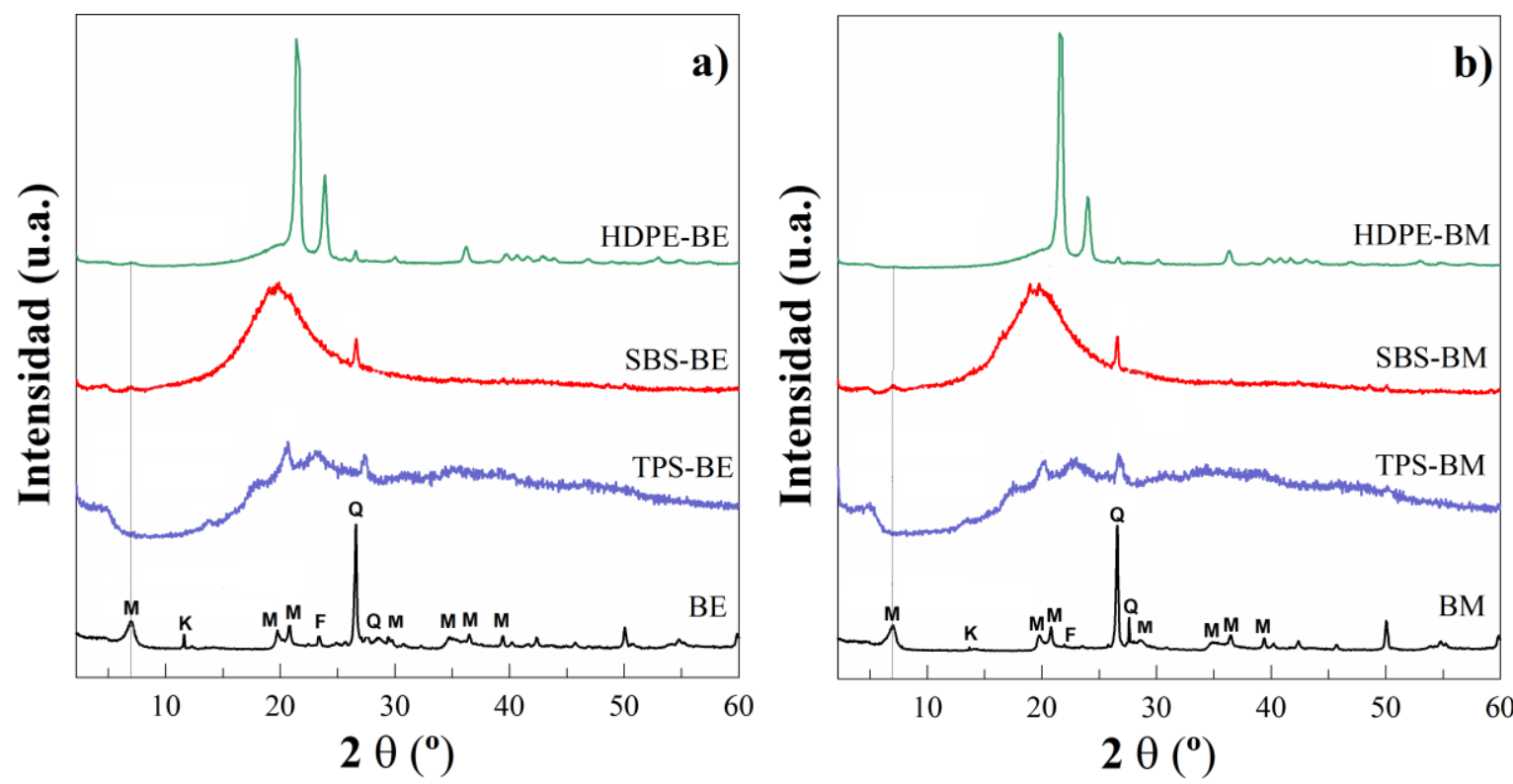

Figura 4: Difractogramas de bentonita comercial (BE) y natural (BM) y de los compuestos con almidón termoplástico (TPS), polietileno de alta densidad (HDPE) y copolímero tribloque de poli-(estireno- $b$-butadieno- $b$-estireno) (SBS).

Los difractogramas de $\mathrm{BE}$ y $\mathrm{BM}$ demostraron que estos minerales están compuestos básicamente por montmorillonita (M). La reflexión del plano basal (001) de montmorillonita para BE y BM, identificada en la Figura 4 para ambas bentonitas, se detectó a $2 \Theta=7,1^{\circ}\left(d_{001}=12,4 \AA\right)$. Asimismo, se observó la presencia de otros minerales adicionales como cuarzo $(\mathrm{Q})$, kaolinita $(\mathrm{K})$ y feldespato $(\mathrm{F})$.

El espectro XRD de los compuestos de TPS-BE/BM muestra que la morfología cristalina del TPS se corresponde con la estructura hidratada tipo V, reportada en trabajos previos [32, 33]. Además, la presencia de las partículas minerales se evidenció por la ocurrencia de las señales típicas de la montmorillonita, componente mayoritario de ambas bentonitas. Para evaluar la eventual intercalación del TPS entre las láminas de ambas bentonitas, se analizó la zona del espectro comprendida entre $2 \Theta=2^{\circ}$ y $2 \Theta=10^{\circ}$ ya que en esta región se encuentra localizada la reflexión del plano (001) de la montmorillonita y el TPS no presenta señales. Por lo tanto, los picos de difracción detectados en esta región espectral sólo pueden ser atribuidos a las partículas de bentonita. Para los compuestos TPS-BE/BM se observó un corrimiento del plano de reflexión (001) de la montmorillonita hacia menores ángulos $\left(\sim 2^{\circ}\right)$ y un incremento significativo en el espaciado interlaminar $\left(d_{001}\right)$ de 5,3 $\AA$ y 5,2 $\AA$ para la BE y BM, respectivamente. Estos resultados podrían ser indicativos de que las cadenas de TPS se insertaron entre las láminas de ambas bentonitas, formando así compuestos intercalados. Según PARK et al. [34], el hecho de que no se obtuvieran materiales completamente exfoliados podría atribuirse a la fuerte interacción polar entre los grupos hidroxilos de la matriz de almidón plastificado y aquellos que están presentes en las láminas de silicato de las arcillas. Esta observación está de acuerdo con los resultados reportados por CYRAS et al. [35] para nanocompuestos de almidón/montmorillonita.

Respecto a las películas de HDPE y sus compuestos, se observaron dos picos de difracción característicos de materiales a base de este polímero sintético. Así, se detectaron señales en $2 \Theta=21,6^{\circ}$ y $23,8^{\circ}$, las cuales se corresponden a los planos de reflexión del polietileno (110) y (200), respectivamente. Difractogramas similares fueron reportados por GU et al. [36] en un estudio acerca de las propiedades de cristalización de materiales a base de HDPE. Los espectros XRD de los materiales compuestos HDPE-BE/BM presentaron además los picos correspondientes a las bentonitas empleadas como relleno de esta matriz polimérica. A diferencia de los materiales compuestos a base de TPS-BE/BM, no se detectó la formación de compuestos intercalados ya que el pico correspondiente al plano (001) de la montmorillonita no sufrió corrimiento hacia menores ángulos ni un incremento significativo en el espaciado interlaminar $d_{001}$. La baja compatibilidad de las 
partículas de bentonita con la matriz de HDPE también fue reportada por WANG et al. [37] y está de acuerdo con el estudio de la microestructura mediante SEM descripto anteriormente. Según PEGORETTI et al. [38], es poco probable que las cadenas poliméricas de poliolefinas como el HDPE se inserten entre las láminas de las partículas de arcillas naturales. Estos autores proponen como alternativa para mejorar la adhesión interfacial entre el polietileno apolar y las láminas polares de silicato de estos minerales, el empleo de un agente de compatibilización como el polietileno injertado con anhídrido maleico. Así, se vería favorecido el proceso de intercalación/exfoliación, mejorando el desempeño mecánico de los materiales compuestos a base de polietileno [39-41].

Los difractogramas de las pelìculas de SBS presentaron un pico amorfo alrededor de $2 \Theta=19^{\circ}$, típico de este tipo de matrices elastoméricas. FU et al. [42] reportó una morfología similar para materiales a base de este copolímero tribloque. En los compuestos SBS-BE/BM se detectó la presencia de las partículas de bentonita pero no se observó corrimiento del pico correspondiente al plano (001) de la montmorillonita ni aumento en el $d_{001}$, indicativo de la conformación de una estructura intercalada. Según YU et al. [43], las láminas de montmorillonita, además de su elevado carácter hidrofílico, poseen un espaciado interlaminar muy pequeño, dificultando la intercalación y el descamado de las mismas por las cadenas del SBS. Estos autores proponen el uso de partículas de montmorillonita organofílica cuyos espaciados interlaminares son mayores que los de la arcilla natural. Esto se debe a que los cationes presentes entre las láminas de silicato han sido intercambiados por moléculas orgánicas, modificando así la estructura y el carácter de la arcilla.

\subsection{Propiedades ópticas de las películas}

Las propiedades ópticas de las películas poliméricas condicionan su funcionalidad y determinan sus aplicaciones específicas. En la Figura 5 se muestra el efecto de la adición de las distintas bentonitas sobre la capacidad de barrera a la radiación UV y la opacidad de las películas de las tres matrices estudiadas.
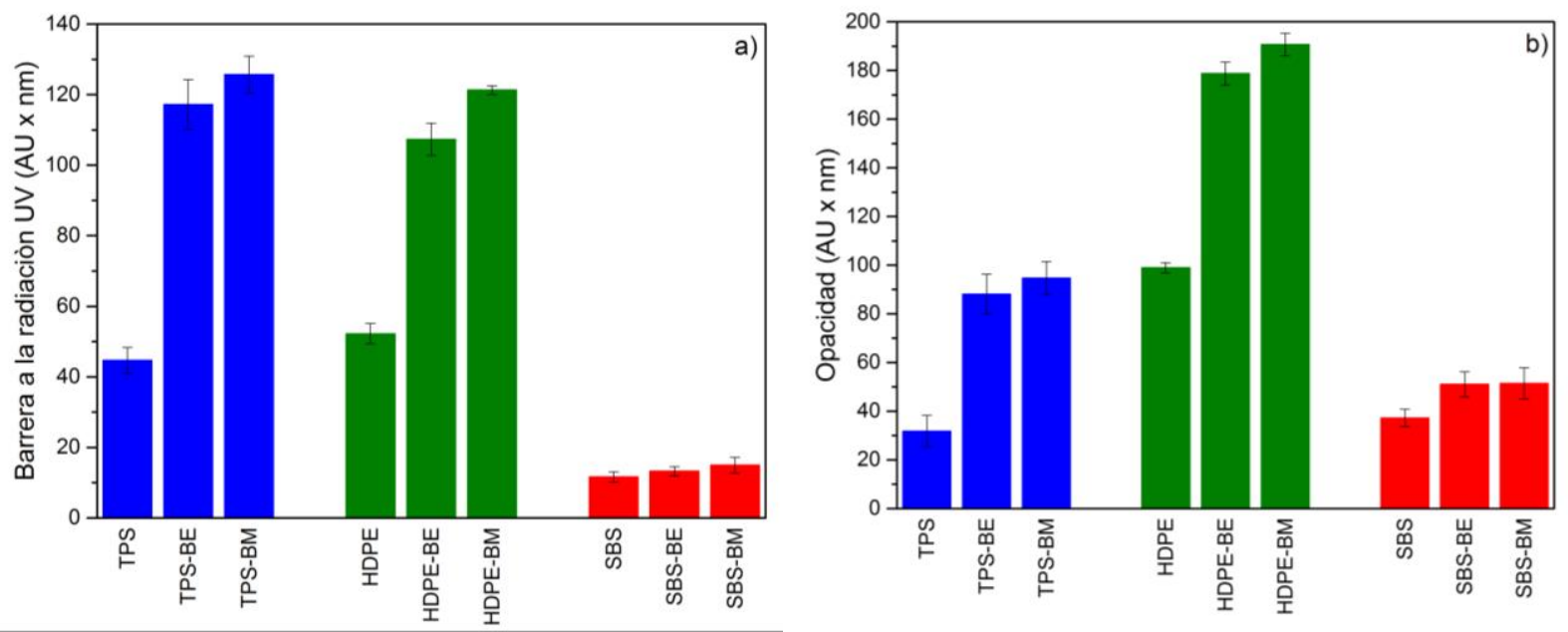

Figura 5: a) Capacidad de barrera a la radiación UV y b) opacidad de compuestos a base de almidón termoplástico (TPS), polietileno de alta densidad (HDPE) y copolímero tribloque de poli-(estireno-b-butadieno-b-estireno) (SBS) y bentonita comercial (BE) ó natural (BM).

La propiedad de barrera a la radiación UV condiciona la aplicabilidad de las películas en el área de envasado de productos alimenticios, farmacéuticos, y cosméticos. Aquellos materiales que eviten el paso de este tipo de radiación podrían emplearse para desarrollar envases para productos susceptibles al deterioro por exposición a la luz UV. En cambio, si las películas poliméricas no actúan como barrera a esta radiación, podrían ser utilizadas como material de envase de productos que, una vez envasados, se sometan a tratamientos de radiación UV para disminuir la carga microbiana de los mismos [32]. Las películas con capacidad de barrera a la luz UV presentan un pico de absorción en la zona espectral de interés. Todos los materiales estudiados presentaron un pico localizado entre 250 y 300 nm, demostrando así su capacidad de absorción de radiación UV. En este sentido, estos materiales serían aptos para ser empleados en desarrollar envases para productos susceptibles a la rancidez oxidativa catalizada por luz UV. Como puede observarse, la presencia de las partículas minerales incrementó significativamente esta propiedad óptica, particularmente para el caso de la matriz de TPS y de HDPE. Si bien la adición de bentonita a las películas de SBS condujo a un leve aumento 
en la capacidad de barrera a la radiación UV, dicho incremento fue menos marcado que en los compuestos basados en TPS y HDPE.

La determinación de la opacidad de los materiales empleados para el desarrollo de envases es relevante ya que esta propiedad óptica influye en la aceptabilidad del producto envasado por parte del consumidor.

En el caso particular de materiales compuestos, se pretende que la incorporación de los diferentes agentes de relleno no disminuya significativamente la transparencia de la matriz. Los cambios en esta propiedad óptica pueden atribuirse a varios factores; el tamaño del relleno, su disposición espacial, como así también a la distribución y dispersión del mismo dentro de la matriz. De acuerdo a lo reportado por KAMPEERAPAPPUN, et al. [43] el valor de opacidad de materiales compuestos que contienen rellenos particulados provee información acerca del tamaño del relleno dispersado en la matriz. Así, partículas más grandes que la longitud de onda de la luz visible podrían obstruir el paso de la misma y dar lugar a materiales compuestos opacos. Como puede observarse en la Figura 5b, la adición de las partículas de bentonita incrementaron significativamente la opacidad de las películas de TPS y HDPE. Como en el caso de la capacidad de barrera a la radiación UV, el cambio en la opacidad de la matriz de SBS por la presencia de estos minerales no resultó tan sustancial.

El hecho de que la adición de bentonita comercial y enológica indujera aumentos en las propiedades ópticas de las tres matrices estudiadas puede atribuirse a que las partículas minerales actúan como barrera física al paso de la luz UV-vis. MBEY et al. [44] reportaron el mismo efecto bloqueante del paso de la radiación ejercido por partículas de caolinita incorporadas a una matriz de almidón de mandioca. Respecto a compuestos a base de polietileno y partículas de arcilla, PENEVA et al. [45] informaron que la incorporación del mineral no afectó significativamente la transparencia de las películas. Estos autores atribuyeron el resultado obtenido al uso de partículas de tamaño nanométrico lo cual permitió una buena dispersión de las mismas en la matriz sintética. Por otra parte, WU et al. [46] estudiaron diferentes matrices elastoméricas, incluyendo materiales compuestos a base de SBS, y reportaron que los mismos presentan uniformidad en la transparencia óptica debido a que los agentes de relleno incorporados eran muy pequeños y presentaban además una estrecha distribución de tamaño.

Hasta el momento, los trabajos de investigación relacionados con películas poliméricas y recubrimientos, estaban básicamente orientados a la mejora de la performance mecánica de los mismos como así también de sus propiedades de barrera a diferentes gases. Sin embargo, en la actualidad el estudio de este tipo de materiales también contempla la evaluación del color de los mismos ya que esta propiedad óptica influye directamente en la apariencia del producto final y en la aceptación del mismo por parte del consumidor [45, 46]. En la Tabla 1 se presentan los valores de luminosidad $(L)$ y de los parámetros de cromaticidad $(a$ y $b$ ) de las películas compuestas estudiadas. Respecto a los valores de luminosidad, se observó que la incorporación de las partículas minerales condujo a una disminución de dicha propiedad óptica en las tres matrices poliméricas estudiadas. De acuerdo con REIS y CANEVAROLO [47], la presencia de arcillas en materiales a base de polipropileno, altera la cristalización de la matriz y la dispersión de la luz entre las fases amorfa y cristalina del polímero y las láminas del mineral, afectando la luminosidad del material compuesto. Por otra parte, como puede observarse en la Tabla 1, la inclusión de partículas de bentonita modificó significativamente los parámetros de cromaticidad de las películas de TPS, HDPE y SBS. Así, se evidenció un incremento tanto en el parámetro $a$ (rojo-verde) como en el $b$ (azul-amarillo). Esto indicaría que la tonalidad de las películas compuestas resultó más verdosa-amarillenta que la de las matrices sin carga. Según YOON et al. [48], el desarrollo de color de los materiales poliméricos con partículas minerales es función del grado de exfoliación de las láminas de arcilla y de las condiciones de procesamiento de los mismos. Por otra parte, FORNES et al. [49] afirman que el color de los compuestos obtenidos por mezclado en fundido generalmente depende de la tonalidad inherente de las partículas de arcilla. A partir de la incorporación de arcillas naturales a matrices poliméricas, se pueden obtener compuestos con tonalidades marrones y amarillentas debido principalmente a la estructura química del mineral y a la presencia de elementos traza. Según MORGAN et al. [50] es poco probable obtener compuestos incoloros empleando como material de relleno arcillas naturales. Otros factores que también afectan el color de este tipo de materiales es el polímero empleado como matriz, como así también el tratamiento previo que hayan sufrido las partículas minerales [48]. 
Tabla 1: Luminosidad $(L)$ y parámetros de cromaticidad ( $a$ : rojo-verde, $b$ : azul-amarillo) de películas a base de almidón termoplástico (TPS), polietileno de alta densidad (HDPE) y copolímero tribloque poli(estireno- $b$-butadieno- $b$-estireno) (SBS) y partículas de bentonita comercial (BE) y natural (BM).

\begin{tabular}{c|c|c|c|c|c|c|c|c|c}
\hline & TPS & TPS-BE & TPS-BM & HDPE & HDPE-BE & HDPE-BM & SBS & SBS-BE & SBS-BM \\
\hline$L$ & $90,9 \pm 0,10$ & $83,4 \pm 1,23$ & $83,7 \pm 0,39$ & $88,9 \pm 0,16$ & $84,8 \pm 0,67$ & $85,8 \pm 1,88$ & $88,3 \pm 0,17$ & $87,4 \pm 0,87$ & $88,4 \pm 0,16$ \\
\hline$a$ & $-0,77 \pm 0,03$ & $-0,15 \pm 0,01$ & $0,32 \pm 0,05$ & $-0,23 \pm 0,04$ & $-0,16 \pm 0,08$ & $-0.17 \pm 0,03$ & $-1,27 \pm 0,23$ & $-1,11 \pm 0,23$ & $-1,33 \pm 0,13$ \\
\hline$b$ & $2,32 \pm 0,18$ & $7,67 \pm 1,17$ & $5,64 \pm 0,16$ & $0,74 \pm 0,12$ & $4,04 \pm 0,48$ & $2,38 \pm 0,14$ & $3,11 \pm 0,62$ & $5,34 \pm 0,15$ & $3,73 \pm 0,25$ \\
\hline
\end{tabular}

\section{CONCLUSIONES}

Se obtuvieron materiales compuestos a partir del agregado de partículas minerales a dos matrices sintéticas: polietileno de alta densidad (HDPE) y copolímero tribloque poli(estireno-b-butadieno- $b$-estireno) (SBS) y a una matriz biodegradable: almidón de maíz termoplástico (TPS). Como agente de relleno se emplearon bentonitas comercial y natural. Se demostró la factibilidad de procesar los compuestos a partir de la incorporación de 5\% w/w de bentonita mediante mezclado en fundido. Adicionalmente, se obtuvieron películas por termocompresión que resultaron flexibles, homogéneas, y translúcidas, con una leve tonalidad amarillenta. Mediante SEM se observó una buena distribución del relleno en las tres matrices obteniéndose la mejor compatibilidad y adhesión de las partículas con la matriz de TPS. La adición de bentonita modificó las propiedades ópticas de las películas debido al efecto bloqueante de las mismas, lo cual se evidenció en un incremento en la capacidad de barrera a la radiación UV y la opacidad de todos los materiales compuestos estudiados.

\section{AGRADECIMIENTOS}

Los autores agradecen a la Agencia Nacional de Promoción Científica y Técnica (ANPCyT), al Consejo Nacional de Investigaciones Científicas y Técnicas (CONICET, Argentina, PIP 0428) y a la Universidad Nacional del Sur (UNS, Argentina, PGI 24/M135).

\section{BIBLIOGRAFÍA}

[1] BERGAYA, F., LAGALY, G., General Introduction: clays, clay minerals, and clay science, 1 ed, Amsterdam. Elsevier Science, 2013.

[2] CHOO, K.Y., BAI, K., "Effects of bentonite concentration and solution $\mathrm{pH}$ on the rheological properties and long-term stabilities of bentonite suspensions", Applied Clay Science, v. 108, pp. 182-190, Mar. 2015.

[3] MANOHAR, D.M., NOELINE, B.F., ANIRUDHAN, T.S., "Adsorption performance of Al-pillared bentonite clay for the removal of cobalt (II) from aqueous phase", Applied Clay Science, v. 31, n. 3-4, pp. 194206, Nov. 2005.

[4] ABU-JDAYIL, B., "Rheology of sodium and calcium bentonite-water dispersions: effect of electrolytes and aging time", International Journal of Mineral Processing, v. 98, n. 3, pp. 208-213, Mar. 2011.

[5] BHATTACHARYYA, K.G., GUPTA, S.S., "Adsorption of a few heavy metals on natural and modified kaolinite and montmorillonite: a review", Advances in Colloid and Interface Science, v. 140, n. 2, pp. 114131, Ago. 2008.

[6] IŞCI, S., GÜNISTER, E., ECE, Ö.I., et al., "The modification of rheologic properties of clays with PVA effect", Materials Letter, v. 58, n. 12-13, pp. 1975-1978, May. 2004.

[7] BERTAGNOLLI, C., KLEINÜBING, S.J., SILVA, M.G.C., "Preparation and characterization of a Brazilian bentonite clay for removal of copper in porous beds", Applied Clay Science, v. 53, n. 1, pp. 73-79, Jul. 2011.

[8] CAGLAR, B., CUBUK, O., DEMIR, E., et al., "Characterization of AlFe-pillared unye bentonite: a study of the surface acidity and catalytic property", Journal of Molecular Structure, v. 1089, pp. 59-65, Feb. 2015.

[9] LIAO, R., YANG, B., YU, W., et al., "Isothermal cold crystallization kinetics of polylactide/nucleating agents", Journal of Applied Polymer Science, v. 104, pp. 310-317, Ene. 2007.

[10] SCHOONHEYDT, R., JOHNSTON, C., Surface and interface chemistry of clay minerals in: Developments in Clay Science - Handbook of clay science, 1 ed., Amsterdam. Elsevier Science, 2006.

[11] ZHOU, Y., HU, N., ZENG, Y., et al., "Heme-protein-clay films: direct electrochemistry and electrochemical catalysis", Langmuir, v. 18, n. 1, pp. 211-219 Ene. 2002. 
[12] ROTHON, R. N., Particulate Fillers for Polymers, 2 ed., United Kingdom, iSmithers Rapra Publishing, 2002.

[13] ABDALLAH W., YILMAZER U., "Novel thermally stable organo-montmorillonites from phosphonium and imidazolium surfactants", Thermochimica Acta, v. 525, n. 1-2, pp. 129-140, Oct. 2011.

[14] ROHLMANN, C.O., HORST, M.F., QUINZANI, L.M., et al., "Comparative analysis of nanocomposites based on polypropylene and different montmorillonites", European Polymer Journal, v. 44, n. 9, pp. 2749-2760, Sep. 2008.

[15] MAGALHÃES, N.F., ANDRADE, C.T., "Calcium bentonite as reinforcing nanofiller for thermoplastic starch", Journal of the Brazilian Chemical Society, v. 21, n. 2, pp. 202-208, Ene. 2010.

[16] MOUMITA, K., ANIL, K.B., "Polymer nanocomposites from modified clays: Recent advances and chalenges", Progress in Polymer Science, v. 51, pp. 127-187, Oct. 2015.

[17] HAIDER, S., KAUSAR, A., MUHAMMAD, B., "Overview of Various Sorts of Polymer Nanocomposite Reinforced with Layered Silicate", Polymer-Plastics Technology and Engineering, v. 55, n. 7, pp. 723743, Ene. 2016.

[18] MBEY, J.A., THOMAS, F., "Components interactions controlling starch-kaolinite composite films properties", Carbohydrate Polymer, v. 117, pp. 739-745, Ene. 2015.

[19] PIERMARÍA, J., BOSCH, A., PINOTTI, A., et al., "Kefiran films plasticized with sugars and polyols: water vapor barrier and mechanical properties in relation to their microstructure analyzed by ATR/FT-IR spectroscopy", Food Hydrocolloids, v. 25, n. 5, pp. 1261-1269, Jul. 2011.

[20] MODABBERI, S., NAMAYANDEH, A., LÓPEZ-GALINDO, A., VISERAS, C., SETTI, M., RANJBARAN, M., "Characterization of Iranian bentonites to be used as pharmaceutical materials", Applied Clay Science, v. 116-117, pp. 193-201, Abr. 2015.

[21] SUN, Z., QU, X., WANG, G., ZHENG, S., et al., "Removal characteristics of ammonium nitrogen from wastewater by modified Ca-bentonites", Applied Clay Science v. 107, pp. 46-51, Abr. 2015.

[22] DING, M., ZUO, S., QI, C., "Preparation and characterization of novel composite AlCr-pillared clays and preliminary investigation for benzene adsorption", Applied Clay Science, v. 115, pp. 9-16, Jul. 2015.

[23] NASWIR, M., ARITA, S., MARSI, S., "Characterization of bentonite by XRD and SEM-EDS and use to increase $\mathrm{pH}$ and color removal, $\mathrm{Fe}$ and organic substances in peat water",

study on the effect of bentonite or feldspar filled low-density polyethylene / thermoplastic Journal of Clean Energy Technologies, v. 1, n. 4, pp. 13-17, Oct. 2013.

[24] PRAMANIK, S., DAS, G., KARAK, N., "Facile preparation of polyaniline nanofibers modified bentonite nanohybrid for gas sensor application”, RSC Advances, v. 3, n. 14, pp. 4574-4581, Feb. 2013.

[25] DE MELO, C., SALOMAO, G.P., EIRAS, G.M., et al., "Properties of extruded xanthan-starch-clay nanocomposites films", Brazilian Archives of Biology and Technology, v. 54, n. 6, pp. 1223-1333, Dic. 2011.

[26] SARIFUDDIN, N., ISMAIL, H., "Comparative sago starch / kenaf core fiber composites", BioResources, v. 8, n. 3, pp. 4238-4257, May. 2013.

[27] SUHAIDA, S.I., ISMAIL, H., PALANIANDY, S., "Study of the effect of different shapes of ultrafine silica as fillers in natural rubber compounds", Polymer Testing, v. 30, n. 2, pp. 251-259, Abr. 2011.

[28] ISMAIL, H., MATHIALAGAN, M., "Comparative study on the effect of partial replacement of silica or calcium carbonate by bentonite on the properties of EPDM composites", Polymer Testing, v. 31, n. 2, pp. 199-208, Abr. 2012.

[29] LIETZ, S., YANG, J.L., BOSCH, E., et al., "Improvement of the mechanical properties and creep resistance of SBS block copolymers by nanoclay fillers", Macromolecular Materials and Engineering, v. 292, pp. 23-32, Ene. 2007.

[30] LIAO, M., ZHU, J., XU, H., et al., "Preparation and structure and mechanical properties of poly (styrene-b-butadiene)/clay nanocomposites", Journal of Applied Polymer Science, v. 92, pp. 3430-3434, Ene. 2004.

[31] PEDRONI, L.G., ARAUJO, J.R., FELISBERTI, M.I., et al, "Nanocomposites based on MWCNT and styrene-butadiene-styrene block copolymers: effect of the preparation method on dispersion and polymerfiller interactions", Composites Science and Technology, v. 72, n. 13, pp. 1487-1492, Ago. 2002.

[32] CASTILLO, L., LÓPEZ, O., LÓPEZ, C., et al., "Thermoplastic starch films reinforced with talc nanoparticles", Carbohydrate Polymers, v. 95, pp. 664-674, Mar. 2013. 
[33] NINAGO, M.D., LÓPEZ, O.V., LENCINA, M.M.S., et al., "Enhancement of thermoplastic starch final properties by blending with poly(E-caprolactone)", Carbohydrate Polymers, v. 134, pp. 205-212, Ago. 2015.

[34] PARK, H., LEE, S., CHOWDHURY, S.R., KANG, T., et al., "Tensile properties, morphology, and biodegradability of blends of starch with various thermoplastics”, Journal of Applied Polymer Science, v.86, pp. 2907-2915, Abr. 2002.

[35] CYRAS, V.P., MANFREDI, L.B., TON-THAT, M.T., et al., "Physical and mechanical properties of thermoplastic starch/montmorillonite nanocomposite films", Carbohydrate Polymers, v. 73, n. 1, pp. 55-63, Jul. 2008.

[36] GU, J., XU, H., WU, C., "Thermal and Crystallization Properties of HDPE and HDPE/PP Blends Modified with DCP”, Advances in Polymer Technology, v. 33, n. 1, pp. 1-5, Oct. 2013.

[37] WANG, S., HU, Y., TANG, Y., et al., "Preparation of polyethylene-clay nanocomposites directly from $\mathrm{Na}+$ montmorillonite by a melt intercalation method", Journal of Applied Polymer Science, v. 89, pp. 25832585, Jun. 2003

[38] PEGORETTI, A., DORIGATO, A., PENATI, A., "Tensile mechanical response of polyethylene - clay nanocomposites", eXPRESS Polymer Letters, v. 1, n. 3, pp. 123-131, Feb. 2007.

[39] LU, H., HU, Y., XIAO, J., et al., "The influence of irradiation on morphology evolution and flammability properties of maleated polyethylene/clay nanocomposite", Materials Letters, v. 59, pp. 648-651, Nov. 2005.

[40] OSMAN, M. A., RUPP, J.E.P., SUTER, U.W., "Effect of non-ionic surfactants on the exfoliation and properties of polyethylene-layered silicate nanocomposites", Polymer, v. 46, n. 19, pp. 8202-8209, Sep. 2005.

[41] CHRISSOPOLOU, K., ALTINTZI, I., ANASTASIADIS, S.H., et al., "Controlling the miscibility of polyethylene/layered silicate nanocomposites by altering the polymer/surface interactions", Polymer, v. 46, n. 26, pp. 12440-12451, Dic. 2005.

[42] FU, B., LEE, A., HADDAD, T., "Styrene-Butadiene-Styrene Triblock Copolymers Modified with Polyhedral Oligomeric Silsesquioxanes”, Macromolecules, v.37, pp. 5211-5218, Abr. 2004.

[43] KAMPEERAPAPPUN, P., AHT-ONG, D., PENTRAKOON, D., et al., "Preparation of cassava starch/montmorillonite composite film”, Carbohydrate Polymers, v. 67, pp. 155-163, Ene. 2007.

[44] MBEY, J.A., HOPPE, S., THOMAS, F., "Cassava starch-kaolinite composite film. Effect of clay content and clay modification on film properties", Carbohydrate Polymers, v. 88, n. 1, pp. 213-222, Mar. 2012.

[45] PENEVA, Y., TASHEV, E., MINKOVA, L., "Flammability, microhardness and transparency of nanocomposites based on functionalized polyethylenes", European Polymer Journal, v. 42, pp. 2228-2235, Ago. 2006.

[46] WU, J., LI, C., WU, Y., et al., "Thermal resistance and dynamic damping properties of poly (styrenebutadiene-styrene)/thermoplastic polyurethane composites elastomer material",Composites Science and Technology, v.70, pp. 1258-1264, Abr. 2010.

[47] REIS, K., CANEVAROLO, S., "Evaluation of the Structure of Polypropylene/ Montmorillonite Nanocomposite by In-Line Light Extinction and Color Measurements During Multiple Extrusions", Polymer Engineering and Science, v. 52, n. 8, pp. 1784-1794, Ago. 2012.

[48] YOON, P., HUNTER, D., PAUL, D., "Polycarbonate nanocomposites: Part 2. Degradation and color formation”, Polymer, v. 44 pp. 7545-7556, Jun. 2003.

[49] FORNES, T., YOON, P., PAUL, D., "Polymer matrix degradation and color formation in melt processed nylon 6/clay nanocomposites”, Polymer, v. 44, n. 24, pp. 7545-7556, Sep. 2003.

[50] MORGAN, A., CHU, L., HARRIS, J., "A flammability performance comparison between synthetic and natural clays in polystyrene nanocomposites", Fire and Materials, v. 29, n. 4, pp. 213-229, Jul. 2005. 